\title{
In-situ TEM Study on Solar Cell
}

\section{Litao Sun}

School of Electronic Science and Engineering, Southeast University, Nanjing, Jiangsu, China (People's Republic)

Energy is an important material guarantee to meet the basic needs of human society and sustainable development. Due to the non-reproducibility of fossil energy and the global attention to the greenhouse effect, the effective use of solar energy is becoming the primary choice in most countries. Quantum dot solar cells have many advantages, such as low preparation cost, adjustable band gap and high theoretical conversion efficiency, and thus have great application potential in the field of solar energy conversion. However, the photoelectric conversion efficiency is still far lower than the theoretical conversion efficiency. It is urgent to explore the basic mechanism of low efficiency at the micro scale and provide guidance for the design of solar cells with high conversion efficiency. This problem poses a theoretical and technical challenge to modern research methods.

To solve this problem, we developed a new type of in situ photoelectric technique inside microscopy independently. Based on this technique, the smallest scale quantum dots heterojunction solar cell structure in the world (including only a nanowire, quantum dots and the electrode) was firstly constructed in the current status. Under the light field, atomic scale resolution of material structure and in situ measurement of photocurrent device at the precision of picoampere level can be realized at the same time. The conversion efficiency of the cell is greatly improved by adjusting the interface area, which reveals that the interface engineering plays an important role on improving the conversion efficiency of the solar cell. The research results are helpful to better understand the internal mechanism of high conversion efficiency of quantum dot heterojunction solar cells and promote the research and optimization design of high conversion efficiency solar cells and the other related photoelectric devices. At the same time, the results provide the best experimental conditions for the direct study of nanoscale devices and promote the rapid development of in situ electron microscopy.

\section{References}

[1] J. R-Manzo, M. Terrones, et.al., Nature Nanotechnology 2, 307 (2007)

[2] X. Liu, T. Xu, et al., Nature Communications 4, 1776 (2013)

[3] X. Guo, G. Fang, et al., Science 344, 616 (2014)

[4] J. Sun, L. He, et al., Nature Materials 13, 1007 (2014)

[5] Q. Zhang, K. Yin, et al., Nature Communications 8, 14889 (2017)

[6] C. Zhu, S. Liang, et al., Nature Communications 9, 421 (2018)

[7] H. Dong, F. Xu, et al., Nature Nanotechnology 14, 950 (2019) 